\title{
Environment-Dependent Breakage Rates in Ball Milling
}

\author{
Rohit Verma \\ Comminution Center, 306 Browning Building, University of Utah, Salt Lake City, UT 84112, USA \\ Ra K. Rajamani \\ Comminution Center, 306 Browning Building, University of Utah, Salt Lake City, UT 84112, USA
}

Breakage rates of particles in a ball mill change with instantaneous particle size distribution in the mill. Slurry density and the presence of a grinding aid also affect breakage rates substantially. The effect of these variables, which constitute the mill environment, on breakage rates has been quantified with a unique estimation method known as the G-H method. This method enables the estimation of breakage rates of all size intervals by a simple linear graphical scheme. In general, breakage rates of coarse particles increase in the presence of fine particles, while the rates of fine particles remain relatively unaffected. Grinding aid restores the fluidity of the solid-liquid mixture even at very high percent solids and so the rate of grinding returns to 'normal' from 'erratic' behavior.

\section{Introduction}

Over the past thirty years or so, there has been a fairly extensive amount of work directed towards ascertaining whether the breakage rate in a grinding mill is dependent on the mill environment. In the past, several investigators showed that, when the operating conditions in a dry batch mill are held constant, the breakage rate function of the feed particles remains unchanged over relatively long grinding times [1--4]. In this paper, we question this 'independence' of breakage rates and explicitly show that they are dependent on mill environment. Specifically, we show the changes in breakage rates for all size intervals as particle size distribution changes during grinding. Recent articles by Gupta [5,6] and Fuerstenau and Abouzeid [7] also questioned the 'independence' of breakage rate with respect to mill environment. However, these papers examine the kinetics of breakage of the coarsest size fraction only, whereas we provide experimental evidence of mill environment-dependent breakage rates for all size intervals. It is well known that the breakage rates are dependent on the mill environment in wet grinding. For example, Tangsathitkulchai and Austin [8,9] and Austin et al. [10] analyzed the influence of slurry density on the breakage parameters of quartz in a laboratory ball mill, and concluded that, in wet batch milling, the breakage of larger particles can accelerate, decelerate or remain constant, depending on loading conditions, slurry 
density and feed size distribution. Klimpel $[11,12]$ determined both acceleration and the slowing down of breakage in experiments under different operating conditions by a radioactive tracer measurement technique.

Past research has made inroads into wet batch milling, but a quantitative model has not been proven yet. It is desirable to know precisely the entire set of breakage rates for a given distribution of particles in the mill. The papers cited above and several others either examined the breakage rates of the coarse size interval only, and/or assumed some functional form for the breakage rates during estimation. Because functional forms do not have an explicit dependence on grinding time, or on size distribution in the mill, they can be applied only for a short duration of grinding time during which the breakage rates can be presumed constant. Hence, breakage rates must be estimated without the imposition of functional forms, as is presented in this paper.

The effect of grinding additives on breakage rates is also shown here. The terms 'grinding aid' or 'grinding additive' refer to a chemical substance that causes a change in the rate of size reduction when mixed into the mill contents. Klimpel and co-workers $[10,13,14]$ show results of grinding experiments in the presence of additives; however, no quantitative explanation has yet been offered that explains or predicts the general behavior of additives. The experimental results presented in this paper show how the breakage rates for all size intervals change in the presence and absence of the grinding additive. Hopefully, these experimental results will help us to understand the modifications in rate of breakage and transport of charge in the presence of grinding additive.

First, a brief description of batch grinding model is presented. Secondly, experimental results for dry and wet grinding as well as with additives are presented. Thirdly, some explanations and possible reasons for the changes in breakage rates as mill environment changes are given. Fourthly, the statistical error analysis of estimates is presented.

\section{Batch Grinding Model}

The formulation of the population balance model and subsequent discussions about its characteristics have been presented by several authors. For a detailed discussion of these models, the reader is referred to a recent review paper [15]. The well-known batch grinding equation, also known as the linear population balance model, is obtained by balancing the mass of material entering and leaving the $i$ th size interval which yields:

$$
\frac{d m_{i}(t)}{d t}=-K_{i} m_{i}(t)+\sum_{j=1}^{i-1} b_{i j} K_{i} m_{i}(t) i=1,2,3, \ldots, n
$$

Here, as in many other publications, the first size interval denotes the top or coarsest size interval with progressively smaller intervals denoted by higher numbers. The exact analytical solution of the batch grinding equation is well known [16]. This solution is nonlinear in breakage rate $\left(K_{i}\right)$ and breakage distribution $\left(b_{i j}\right)$ parameters, so either the optimization or the root finding technique is used to estimate parameters. Alternately, the set of $n$ differential 
equations can be conveniently represented by a single matrix equation and the analytical solution can be fitted by nonlinear methods to experimental data, thus estimating $K$ and $B$ values $[17,18]$.

For a total of ' $n$ ' size intervals, the above model uses $(n-1)$ breakage rate parameters and $(n-1)(n-2) / 2$ breakage distribution parameters. Such a large parameter set presents problems in determining the values of these parameters from a limited set of grinding data. Hence, different functional forms for the breakage rates and breakage distributions have been proposed by several researchers. One such functional form is given as

$$
K_{i}=K_{1}\left(\frac{X_{i} X_{i+1}}{X_{1} X_{2}}\right)^{\zeta}
$$

And

$$
b_{i j}=\alpha_{1}\left(\frac{x_{i}}{x_{j+1}}\right)^{\alpha 2}+\left(1-\alpha_{1}\right)\left(\frac{x_{i}}{X_{j+1}}\right)^{\alpha 3}
$$

Here the parameters $K_{1}, \zeta, a_{1}, a_{2}$ and $a_{3}$ are numerically estimated from experimental data [18]. Because direct measurement of breakage rate and breakage distribution parameters is possible for the top or coarsest size only, past works have centered on the back-calculation methods, which use the complete size distribution data generated at several grinding times. The functional forms reduce the number of parameters in the estimation scheme to a maximum of five. However, the explicit dependence of the parameters on the mill environment is unknown. Furthermore, breakage rates estimated by using functional forms do not have any information about how the rates change with grinding time, fineness of the charge or other operating characteristics. In fact, the functional forms obscure the effects due to mill environment. Hence, a newer method which does not impose any functional form is needed for estimating breakage parameters. Such a method published by Kapur and co-workers, known as the G-H solution, enables a fresh look at the batch grinding process [19-21].

The $\mathrm{G}-\mathrm{H}$ solution is an approximate solution to the size-discretized batch grinding equation. This solution lends itself to easy evaluation of $K$ and $B$ parameters graphically from the experimental data. Rajamani and Guo [22] modified the G-H solution to estimate breakage distribution and time-dependent breakage rates. The $\mathrm{G}-\mathrm{H}$ solution scheme proposes an approximate solution to the batch grinding equation (cumulative form) in the following manner $[19,20]$ :

$$
\frac{1}{t} \ln \frac{R_{i}(t)}{R_{i}(0)}=G_{i}+H_{i} \frac{t}{2}
$$

where $G_{i}$ and $H_{i}$ are functions of the cumulative mass fraction retained $\left(R_{i}\right)$, breakage rate $\left(K_{i}\right)$ and breakage distribution parameters $\left(B_{i, j}\right)$.

Kapur and co-workers, in a series of papers, have shown that the G-H solution closely approximates the analytical solution over a reasonable length of grinding time, in a batch mill 
operation. In fact, the G-H solution is valid, without loss of any accuracy, until $95 \%$ of the material is broken out of a size interval [20]. The attractive feature of the G-H solution is that the plot of $\ln \left[\frac{R_{i}(t)}{R_{i}(0)}\right] / t$ versus $t$, termed the G-H plot, is a straight line. In other words, if the experimental data exhibit a straight line in such a plot, it confirms that the grinding mill behavior follows the linear population balance model. Purker et al. [21] rearranged the approximate solution of Eq. (4) to determine breakage rates as follows:

$$
\begin{aligned}
& K_{i}(t)=\left[-G_{i}-H_{i} t-K_{i-1} B_{i+1, i-1} \frac{R_{i-1}(t)}{R_{i}(t)}+\sum_{j=1}^{i-2}\left(K_{j+1} B_{i+1, j+1}-K_{j} B_{i+1, j}\right) \frac{R_{j}(t)}{R_{i}(t)}\right] \times \\
& {\left[1-\frac{R_{i-1}(t)}{R_{i}(t)}\right]^{-1}}
\end{aligned}
$$

The right-hand side of Eq. (5) contains the cumulative mass fraction retained on the bottom size of an interval at a given time. Hence, the calculated breakage rates will change as the size distribution in the mill changes. Accordingly, $K_{i}, G_{i}$ and $H_{i}$ should be written as $K_{i}(t)$, $G_{i}(t)$, and $H_{i}(t)$, respectively. However, for the sake of simplicity, the dependence on time is not indicated hereafter, although it is implied.

With the breakage distribution functions and the product size distributions, the breakage rates for all sizes can be estimated easily by applying the $\mathrm{G}-\mathrm{H}$ solution on successive time intervals. Rajamani and Guo [22] and Verma [23] explain the different steps in the G-H estimation scheme in detail, while it is briefly explained in the following section for the sake of clarity.

\section{Step 1: Estimation of Breakage Distribution Function}

For a monosize feed, it can be shown that

$$
B_{i, 1}=\frac{G_{i}}{-K_{1}}=\frac{G_{i}}{G_{1}}
$$

Eq. (4) is an equation of a straight line. Hence $G_{i}(i=1,2,3 \ldots, n)$ can be easily estimated by the means of standard linear regression. After determining breakage distribution function values for the top size interval as above, the rest of the values are obtained with the normalizability assumption:

$$
b_{i, j}=b_{i-j+1,1} \text { for } i>j
$$

which is quite valid for homogeneous ores. However, for heterogeneous ores, monosize batch grinding experiments for each individual size should be done to determine $b_{i, j}$ values. In general, the normalizability assumption is invoked even for heterogeneous ores.

\section{Step 2: Calculation of the Top Size Breakage Rate}

For a short interval of grinding time $\left(t_{1}, t_{2}\right), K_{1}$ can be evaluated directly from the top size disappearance plot, i.e.: 


$$
K_{1}=\frac{\ln \left[m_{1}\left(t_{2}\right) / m_{1}\left(t_{1}\right)\right]}{\left(t_{1}-t_{2}\right)}
$$

Step 3: Calculation of $G_{i}$ and $H_{i}$

In time interval $\left(t_{1}, t\right)$ Eq. (4) becomes

$$
\frac{1}{t-t_{1}} \ln \frac{R_{i}(t)}{R_{i}\left(t_{1}\right)}=G_{i}+\frac{H_{i}\left(t-t_{1}\right)}{2}
$$

Eq. (9) is an equation of a straight line and, hence, the slope $H_{i} / 2$ and the intercept $G_{i}$ are determined easily with the grinding data available at times $t_{1}$ and $t$.

Step 4: Cumulative Mass Fraction Retained in Size Interval $I, R_{i}(t)$

The availability of $R_{i}(t)$ in successive time intervals is essential for the evaluation of time dependence of breakage rates. Conducting batch grinding experiments for short time intervals is impractical as material would be lost during unloading and charging the mill. The changes in size distribution after half a minute of grinding time may not be detectable with a set of sieves. Therefore, a method to get a reliable estimate of short-time grinding data from actual batch mill experiments conducted at longer time intervals is needed. Cubic spline interpolation was found to be satisfactory for this purpose [24].

The G-H estimation scheme described by the above four steps is based on an approximate solution to the batch grinding equation. Hence, the effectiveness of the estimation scheme was first verified with simulated data which showed that the mean absolute percentage error in estimating breakage rate and breakage distribution parameters is less than $2 \%[23]$.

\section{Experimental}

Batch dry and wet grinding experiments were done with limestone and copper ore. All of the experiments were carried out in a stainless-steel mill, $25 \mathrm{~cm}$ in diameter and $29 \mathrm{~cm}$ long, fitted with eight rectangular $0.3 \mathrm{~cm}$ high lifters. Typical holdup of solids in this mill is $3.3 \mathrm{~kg}$. All of the experiments were carried out at $54 \mathrm{rpm}$ ( $60 \%$ of mill critical speed) and with $50 \%$ mill volume filled with steel balls. The mill was equipped with a Graham variable-speed transmission and a BLH torque sensor interfaced with a Sargent recorder. The torque sensor enables a record of mill power draft throughout each experiment.

Limestone used in the experiments was acquired from Utah Calcium Co., UT. As received, it was approximately $95 \%$ - 10 mesh particles. Three different types of feed size distributions were used in the experiments. They are monosize (10× 14 mesh), natural size (-10 mesh; $15-20 \%$ material in the top size interval), and coarse size (-10 mesh; $30-35 \%$ material in the top size interval).

Copper ore of sizes below $1.2 \mathrm{~cm}$ was acquired locally. 
First, the ore was crushed by roll crushers to get approximately $95 \%$-10 mesh particles. This material was sieved for $20 \mathrm{~min}$, and $\mathbf{- 1 0}$ mesh particles were retained as natural size feed. Using a combination of dry' and wet sieving, monosize samples were obtained for $10 \times 14,14 \times$ $20,20 \times 28$ and $28 \times 35$ meshes. The $10 \times 14$ mesh particles were dry ground for 1.5 to $2.0 \mathrm{~min}$ to obtain several coarse size (-10 mesh) feeds. XFS4272, frequently mentioned as an efficient grinding additive, was used in some of the experiments. It is a patented chemical additive of the Dow Chemical Co. made up of low molecular weight water-soluble anionic polymer.

\section{Dry Grinding Results}

In the analysis of dry grinding kinetics, limestone was the experimental solid. First, monosize $10 \times 14$ mesh feed was ground separately to determine the feed size breakage distribution function. The G-H method was used to determine the breakage distribution function from monosize batch grinding data. Since limestone is a homogeneous material, the normalizability assumption (Eq. (7)) was invoked to obtain the entire set of breakage distribution functions. Others who have used limestone from the same source also concluded that the breakage distribution function is indeed normalizable [25].

To check the dependence of breakage rates on feed size distributions, two samples (Feed $A$ and Feed $B$ ) were prepared with different size distributions. Feed $A$ had a natural size distribution with approximately $17 \%$ material in the top size interval (10x14 mesh). Feed B was a coarse feed with $31 \%$ material in the top size interval.

Breakage rate variations were analyzed by the G-H scheme. Fig. 1 shows the time dependence of breakage rates for a natural size feed (Feed A). First there is an acceleration followed by deceleration and acceleration of breakage for $10 z 14$ mesh particles. There is a general trend of increase of breakage rates for $10 \times 14$ mesh particles as grinding time increases. Particles in the smaller size intervals also show changes in breakage rates for the first $2 \mathrm{~min}$, but there is not much variation after that. Furthermore, the magnitude of change in breakage rates is smaller for lower particle sizes.

The estimated breakage rates for coarse size feed sample (Feed B) are shown in Fig. 2. Breakage rates for $10 \times 14$ mesh particles increase for the first $2 \mathrm{~min}$, then decrease sharply for the next $1 \mathrm{~min}$ and increase again. All other size fractions show relatively less variation in breakage rates, except at the time when the breakage rate of the top size decreases sharply. Smaller size intervals show an increase in breakage when breakage rates of the top size decrease sharply. Even though the nature of variations in breakage rates is quite different for Feed A (Fig. 1) and Feed B (Fig. 2), it is clear that the breakage rates do depend on particle size distributions or mill environment. The breakage rates for coarse feed are much less than rates for natural size feed. For example, breakage rates for $10 \times 14$ mesh particles increased from 0.6 to $0.9 \mathrm{~min}^{-1}$ in $5 \mathrm{~min}$ for natural size feed, whereas the rate stayed around $0.4 \mathrm{~min}^{-1}$ for coarse feed. This variation in breakage rates, however, is minimal for finer sizes. It appears that the 
'acceleration and deceleration of breakage' effect is more pronounced for the coarser size intervals and the breakage rates of smaller sizes do not change much with grinding time.

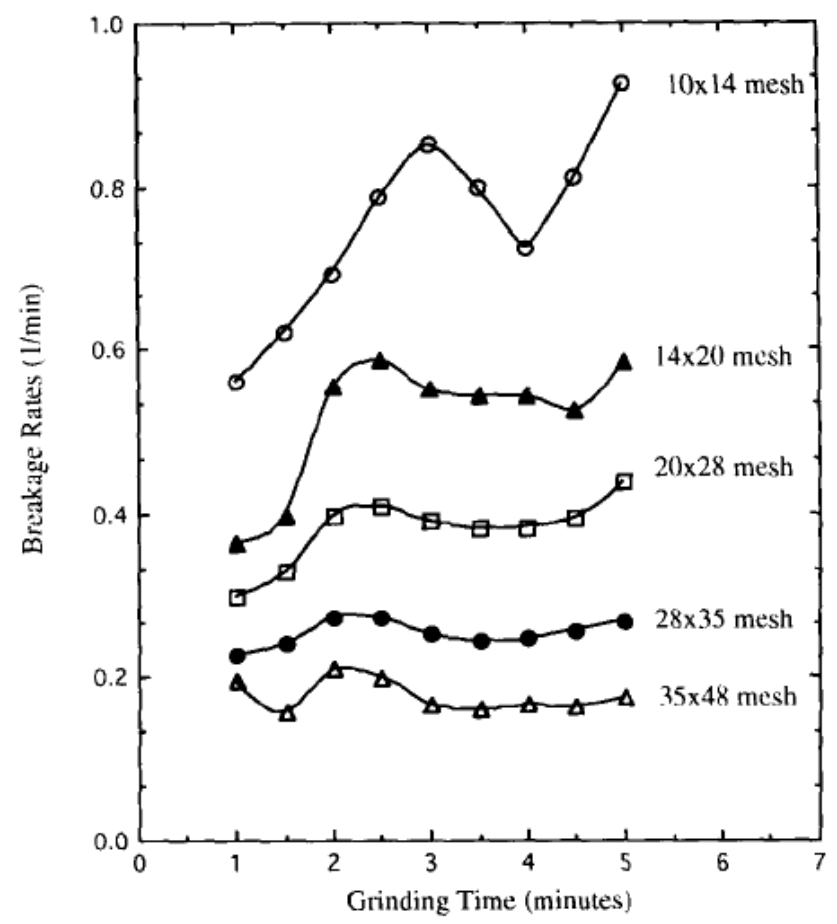

Fig. 1. Time dependence of breakage rates for a limestone dry grinding experiment (Feed A natural size feed).

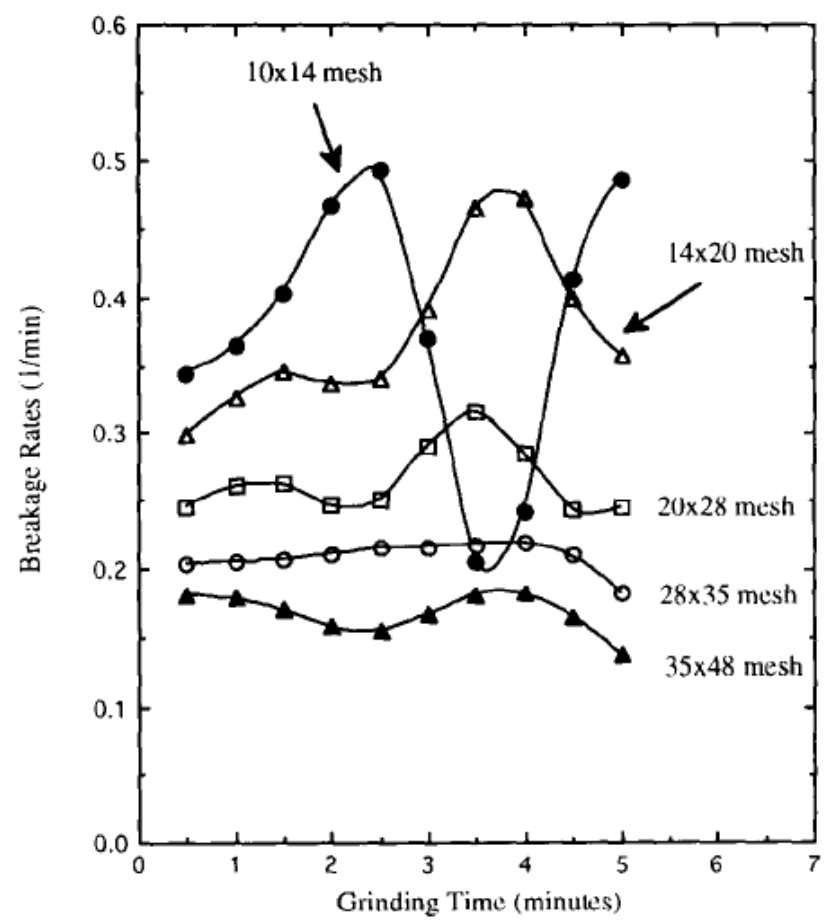

Fig. 2. Time dependence of breakage rates for a limestone dry grinding experiment (Feed Bcourse feed) 
Large variations in the breakage rate for the top size interval in the coarse feed experiment suggest a possibility of experimental error. To eliminate this possibility, the experiment was repeated with nearly the same feed. This feed had $35 \%$ material in the top size interval. Feed B, used in the previous experiment, had 31\% material in the top size interval. Breakage rates estimated with the G-H scheme are shown in Fig. 3. These breakage rates are very similar to those in Fig. 2, confirming that the sharp decrease in breakage rate for the top size interval is not due to experimental errors and is the nature of the material.

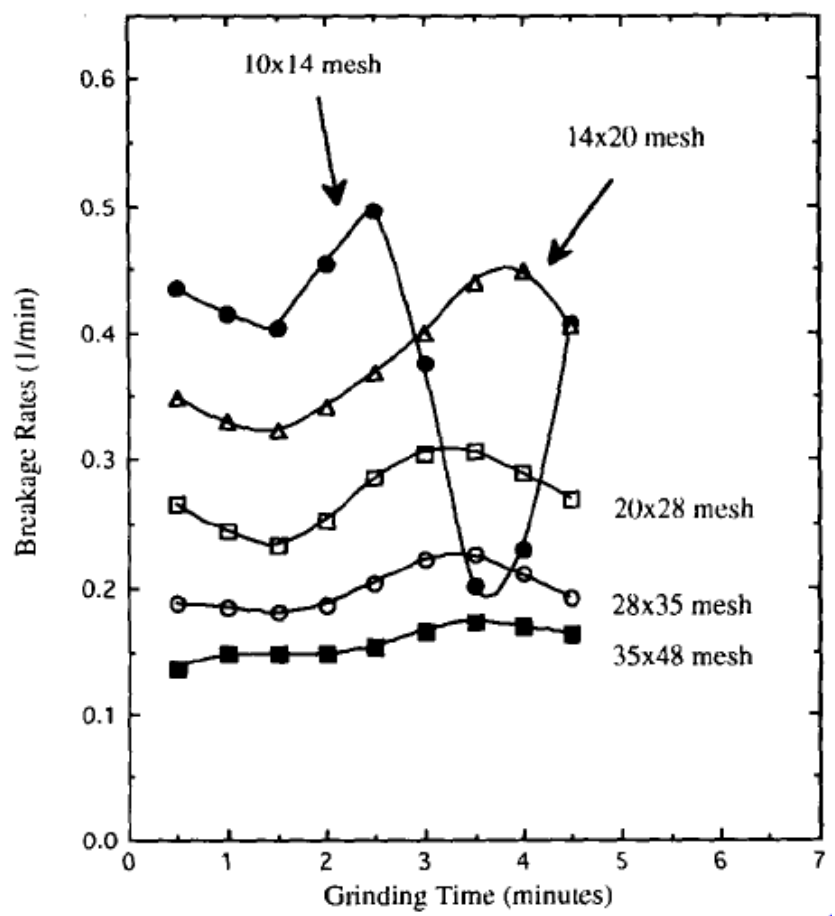

Fig. 3. Time dependence of breakage rates for a limestone dry grinding experiment (course feed, second experiment)

An alternative way of confirming change in breakage rate is by looking at the top size disappearance plot, the slope of which is the breakage rate for the top size interval. Fig. 4 shows top size disappearance plots for the three experiments reported above (Feed A, Feed B and the repeated experiment with coarse feed). It can be clearly seen that the two feeds with nearly the same feed size distributions have almost the same slopes. However, the slopes for the experiment with natural size feed are obviously different from those for coarse feeds. Hence, the natural size feed represents a higher value of breakage rate than coarser feeds. In general, the breakage rates are dependent on the particle size distributions and finer feeds exhibit higher breakage rates.

The top size disappearance plots shown in Fig. 4 are not perfectly straight lines. For instance, the plot for natural size feed shows a gradual increase in slope from 1.0 to $4.0 \mathrm{~min}$, and a slight decrease and increase of slope is seen after that. These slight changes in slope are consistent with the breakage rates estimated by the G-H scheme. Wherever there is a change in 
slope in the top size disappearance plot, a change in the breakage rate is confirmed by the G-H scheme. Disappearance plots for the coarse feeds also show similar changes in slope. Hence, it can be inferred that breakage rates do change with time in batch dry grinding.

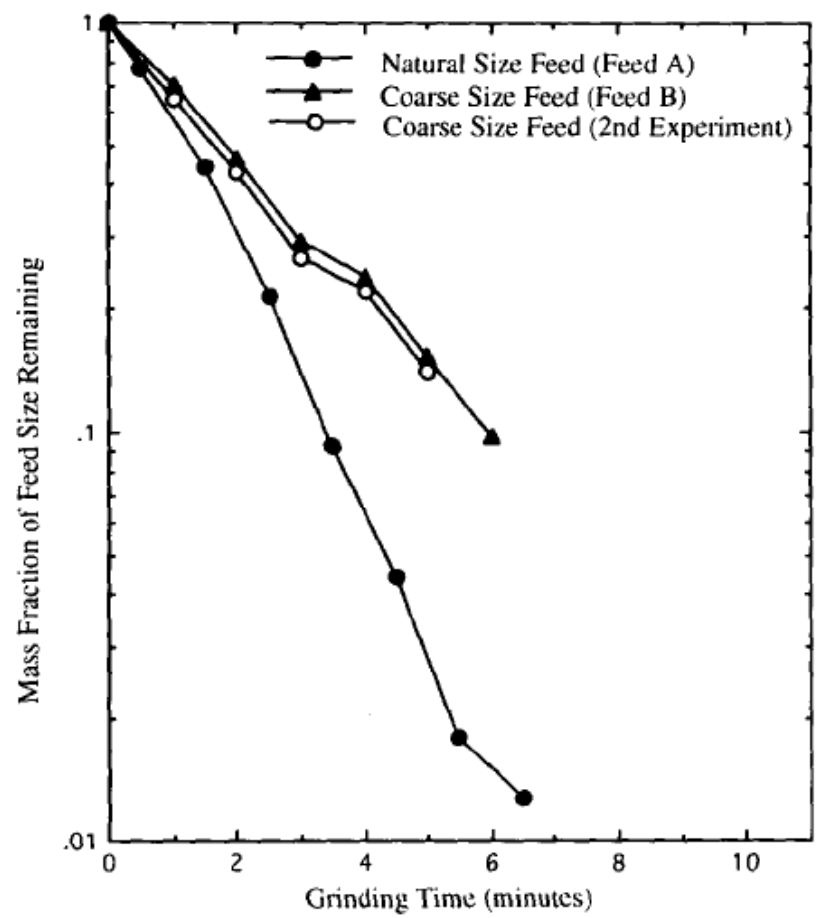

Fig. 4. Top size disappearance plot for limestone dry grinding experiments.

\section{Wet Grinding Results}

The G-H plot (Eq. (4)) of batch grinding data can be used to check the time dependence of breakage rates. Straight lines on this plot indicate constant breakage rates, provided that the same plot for the top size interval exhibits a slope of zero. Such a G-H plot for a wet grinding experiment with natural size limestone (60\% solids) is shown in Fig. 5. For comparison, a set of simulated data, for which the breakage rates were held constant, is also shown. The marked deviations of the experimental data from a straight line confirm that the breakage rates are changing with time. From Fig. 5 it is also clear that top size particles show maximum deviations from linearity. This deviation is negligible for particles smaller than $28 \times 35$ mesh. Hence, it can be concluded that breakage rates of coarse sizes show a large deviation from linearity and vary with time; at the same time, breakage rates of fines do not change much with time.

Next, we turn to the analysis of batch grinding of copper ore which is markedly heterogeneous. First, four different monosize $(10 \times 14,14 \times 20,20 \times 28,28 \times 35$ mesh sizes) grinding experiments were done to obtain the set of non-normalizable breakage distribution functions. Therefore, the $B$ values calculated from the actual experimental data were used for the corresponding four sizes, and the rest of the size intervals were assumed to be normalizable with respect to $28 \times 35$ mesh particles. 


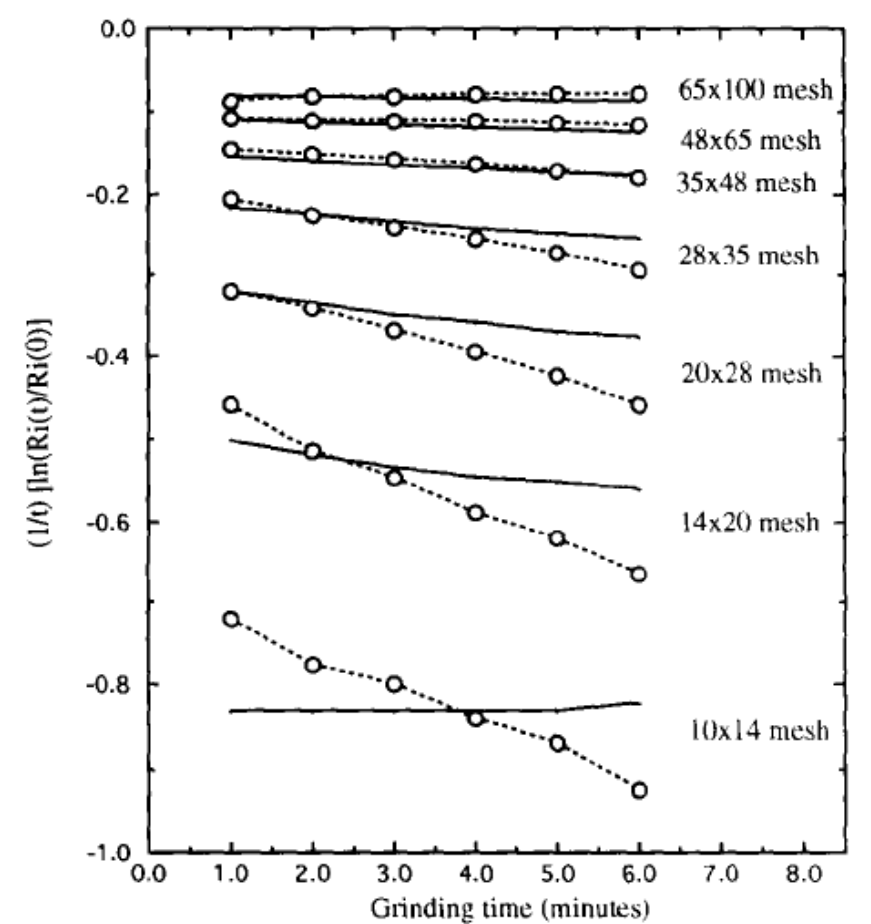

Fig. 5. G-H plot for a limestone wet grinding experiment at $60 \%$ solids: (- - -, experimental data, --- simulated data).

The time-dependent breakage rates for a natural size batch grinding experiment at $60 \%$ solids is shown in Fig. 6 . The breakage rate for the top size particles (10x14 mesh) increases in

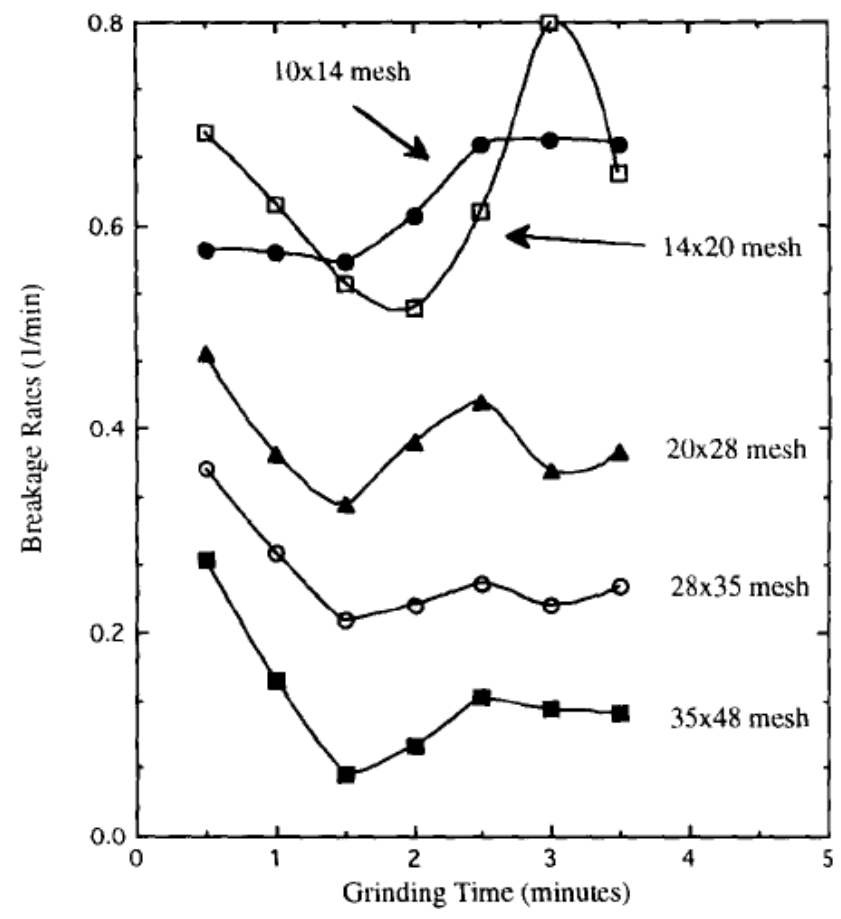

Fig. 6. Time dependence of breakage rates for a copper ore wet grinding experiment at $60 \%$ solids (natural size feed). 
the beginning and then stays constant at a higher value. However, the breakage rates for other smaller sizes decrease, increase, and then attain a steady-state value. This trend is followed by all sizes, but the variations decrease in magnitude with particle size.

To study the dependence of breakage rates on feed size distribution at a fixed slurry concentration, another experiment was done with a coarser feed (31\% material in $10 \times 14$ mesh size interval) at $60 \%$ solids. Breakage rates estimated by the G-H scheme are shown in Fig. 7. The $10 \times 14$ mesh particles show a similar trend to the natural size feed experiment, but other size fractions exhibit a continual acceleration of breakage. It appears that, when batch grinding is started with a coarse feed, fines are generated, and as the fineness of the material inside the mill increases they enhance the breakage of coarse particles. So acceleration of breakage for almost all coarse intervals is noticed. However, when the same experiment was done with a finer natural size feed (shown in Fig. 6), the coarsest sizes exhibited a deceleration of breakage. Here too many fine particles surrounding the coarse particle may have led to a 'cushioning' effect.

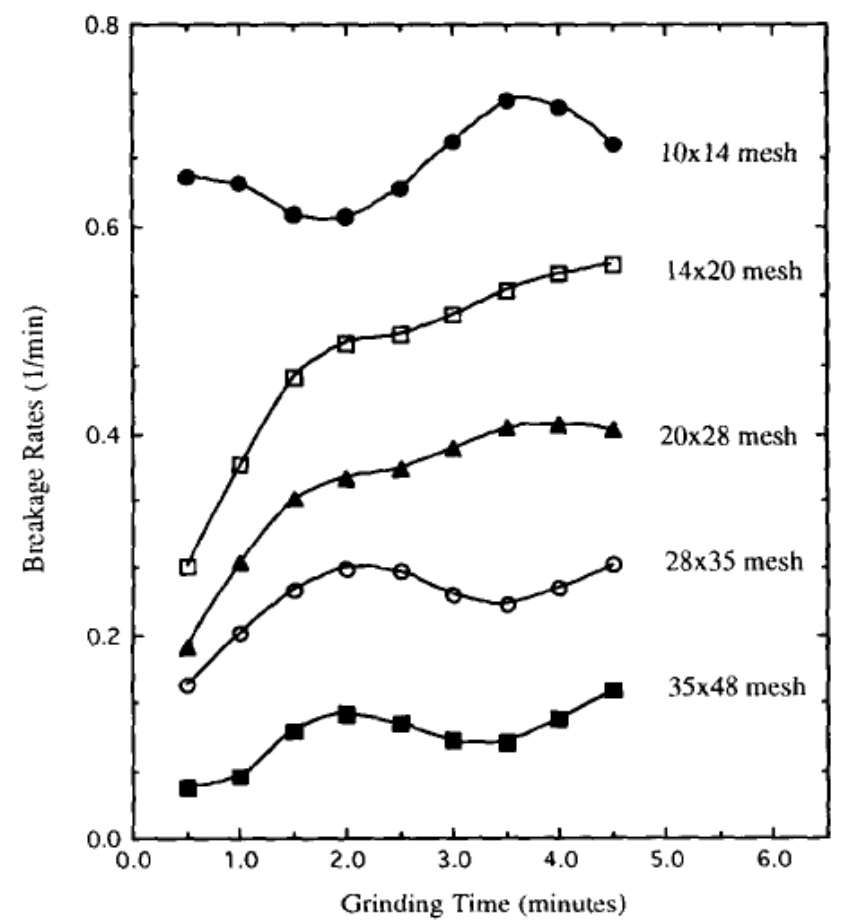

Fig. 7. Time dependence of breakage rates for a copper ore wet grinding experiment at $60 \%$ solids (coarse feed).

Next, several sets of experiments were performed at higher percentage solids $(65 \%$, $75 \%, 80 \%$ and $85 \%$ solids) to study the effect of slurry concentration on breakage rates. Figs. 8 and 9 show time dependence of breakage rates for $65 \%$ and $75 \%$ solids copper ore experiments, respectively. In both these experiments, the trends are the same - most of the size fractions show a decrease in breakage rate followed by an increase. However, these plots are quite different from those for the $60 \%$ solids experiment (Fig. 7). In the $60 \%$ solids 
experiment, the breakage rates for most size intervals decrease with grinding time, but for $65 \%$ and $75 \%$ solids experiments most of the size intervals show a decrease followed by an increase

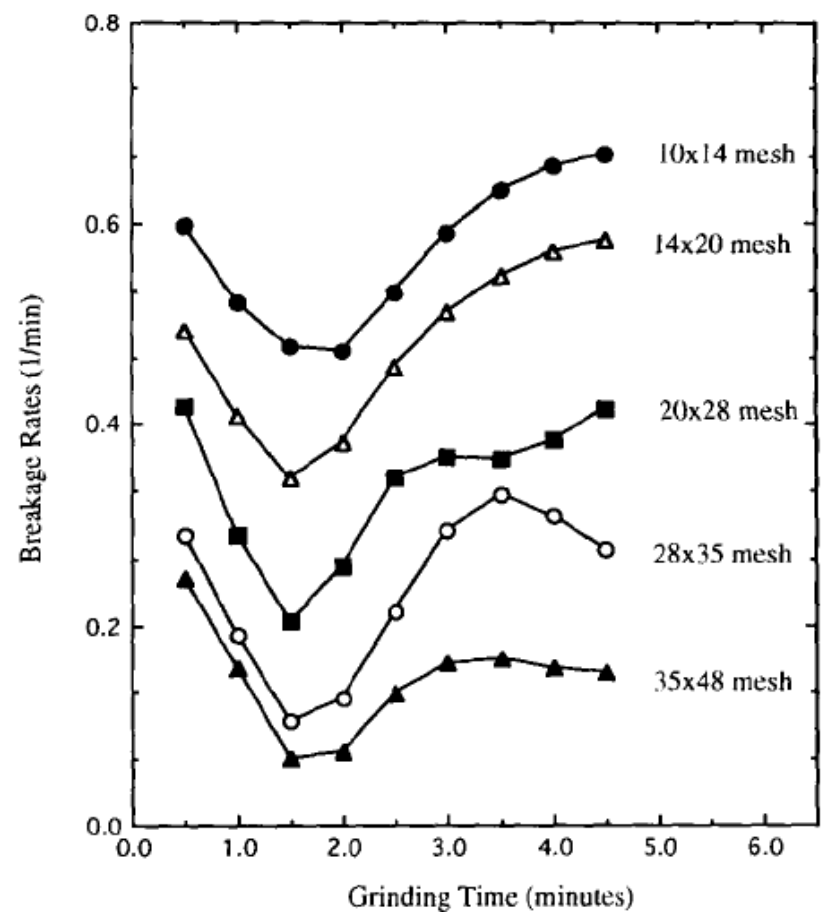

Fig. 8. Time dependence of breakage rates for a copper ore wet grinding experiment at $65 \%$ solids (natural size feed).

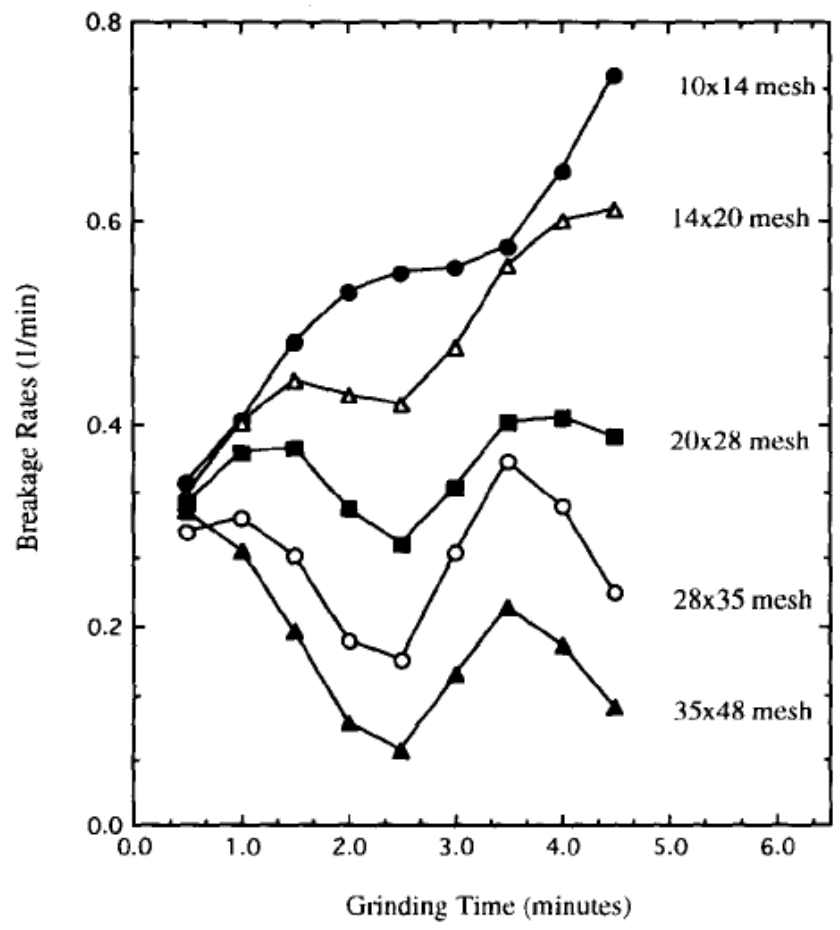

Fig. 9. Time dependence of breakage rates for a copper ore wet grinding experiment at $75 \%$ solids (natural size feed). 
in breakage rates. Although the exact reason for these variations for different slurry densities is not known, these observations do lead to the conclusion that the breakage rates show different trends at different slurry density.

\section{Wet Grinding in the Presence of a Grinding Additive}

At very high concentration of solids, the wetting of solids by water is incomplete and, hence, the grinding process becomes erratic. Batch grinding experiments were conducted at $80 \%$ and $85 \%$ solids to study the effect of grinding additives on breakage rates of particles in different size intervals. The additive dosage was $0.03 \mathrm{wt} . \%$ on the basis of dry solids, as recommended by previous researchers [12]. Again monosize (10X14 mesh) grinding tests were done to determine the breakage distribution functions by the G-H scheme. Next, natural size wet grinding experiments were done at $80 \%$ and $85 \%$ solids without any additive. Fig. 10 shows the breakage rates estimated by the G-H scheme for the $80 \%$ solids experiment without additive. In the $85 \%$ solids experiments a similar erratic pattern was followed by particles in all size classes.

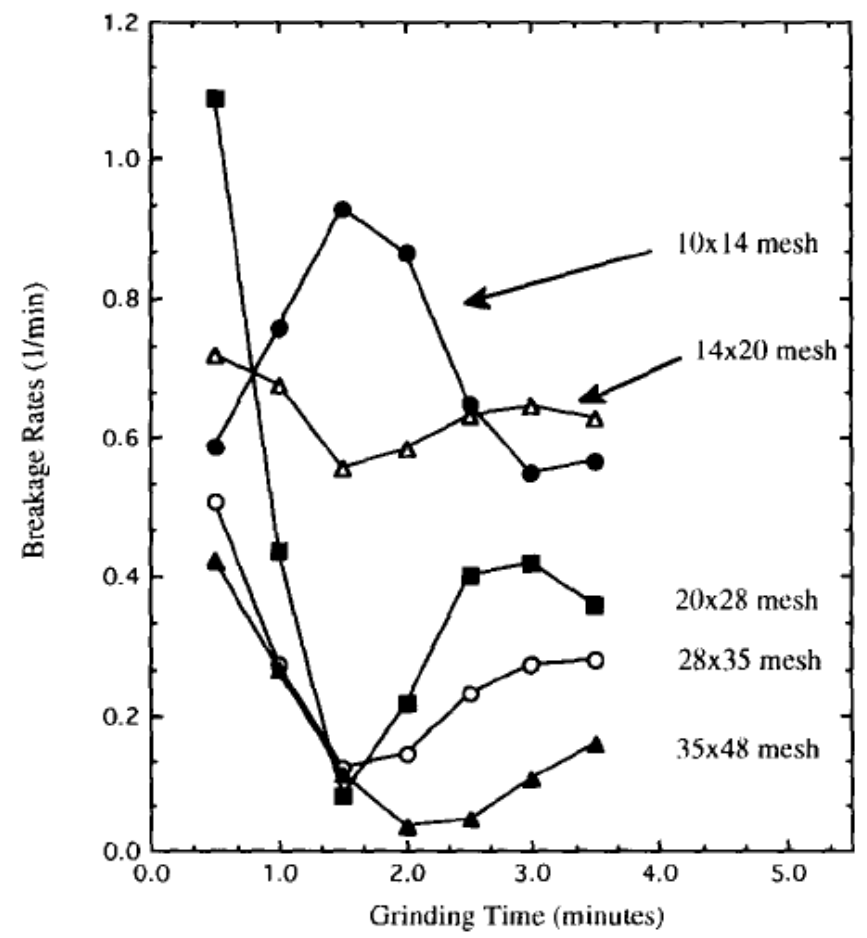

Fig. 10. Time dependence of breakage rates for a limestone wet grinding experiment at $80 \%$ solids (natural size feed).

Even though ball charge motion imposes a constant force regime on particles, the ease of flow of the particles through the balls is equally important for breaking particles. At 80 to $85 \%$ solids, the slurry is so thick that it behaves like separate lumps. In fact, the entire particle mass may not be evenly wetted by such a low addition of water. While opening the mill during experiments, a lot of material was found to stick near the corners and close to the lifter bars. 
The addition of chemical additives makes the slurry less viscous even at high percentage solids. For instance, the limestone mass and the balls were found moving freely in the mill after the addition of XFS4272. During experiments, it was very easy to pour material out of the mill, and only a very small fraction of material was stuck in the corners.

Natural size wet grinding experiments repeated in the presence of XFS4272 show a totally different trend. Fig. 11 shows breakage rates of limestone at $80 \%$ solids in the presence of 0.03 wt.\% XFS4272. Breakage rates in the presence of grinding additive are quite different from the previous experiment where XFS4272 was not added. A more regular pattern is seen in the presence of additive. All size intervals show deceleration, followed by acceleration and again deceleration of breakage. Even the smaller sizes show sharp changes in breakage, unlike in wet experiments without grinding aid.

Fig. 12 shows the results of natural size wet grinding experiment at $85 \%$ solids in the presence of XFS4272. Once again a very different pattern of breakage rates is obtained in the presence of additive. Breakage rates for all different sizes seem to collapse on a single curve.

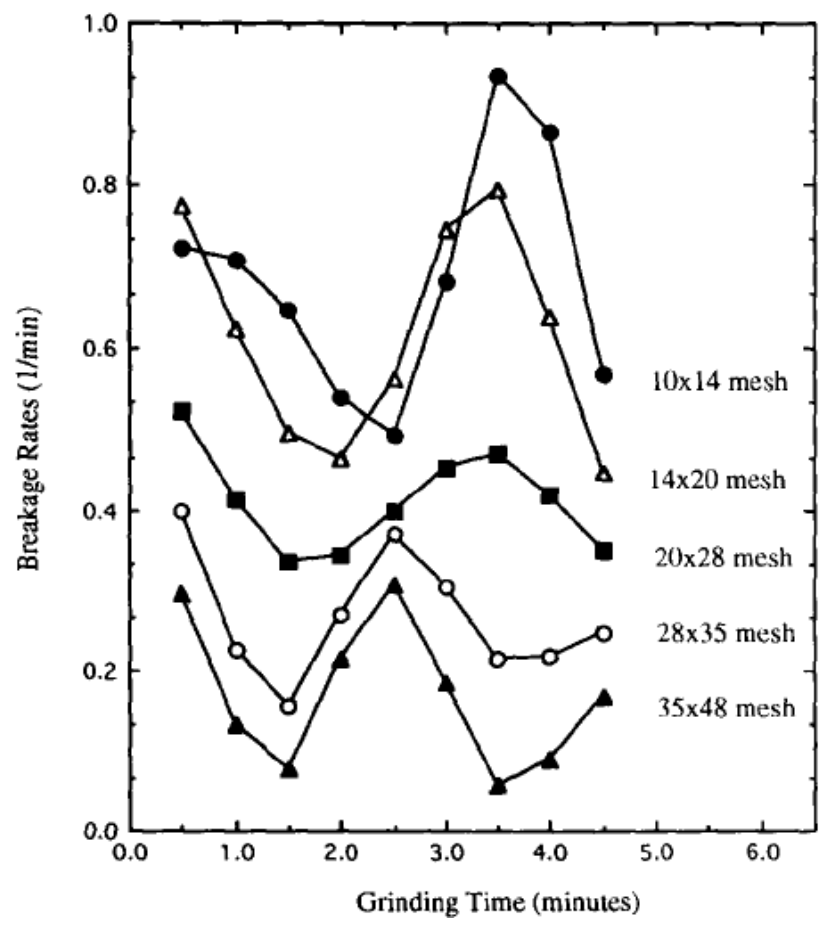

Fig. 11. Time dependence of breakage rates for a limestone wet grinding experiment at $80 \%$ solids (natural size feed); XFS4272 dosage: $0.03 \%$ on dry solids basis.

They all exhibit deceleration, followed by acceleration and again deceleration of breakage. However, the addition of XFS4272 does not seem to affect the breakage rate of $10 \times 14$ mesh particles to such a large extent. Both in the presence and absence of XFS4272, breakage rates of $10 \times 14$ mesh particles stay around 0.2 min-1.

Looking at Figs. 10-12, it can be concluded that the addition of a grinding additive 
changes the breakage rate. However, it cannot be concluded that the breakage rates have substantially increased in the presence of XFS4272.

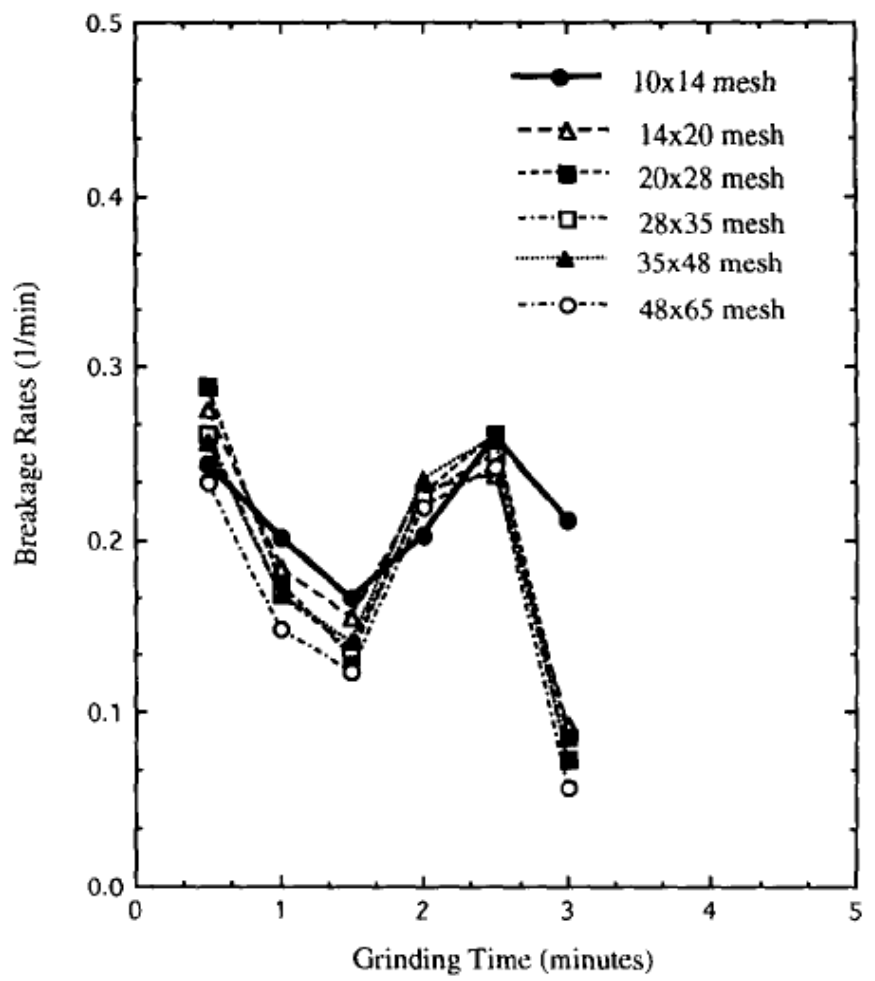

Fig. 12. Time dependence of breakage rates for a limestone wet grinding experiment at $85 \%$ solids (natural size feed); XFS4272 dosage: $0.03 \%$ on dry solids basis.

\section{Statistical Analysis of Batch Grinding Data}

As shown earlier, breakage rates show sharp variations with time under various experimental conditions. Since the variations depend on minor changes in mass fractions held within size intervals, one would like to know how experimental errors affect the estimated values. Due to the complexity of the G-H estimation expression, an analytic examination of estimation errors is impossible and so a statistical procedure was used to determine the bounds on estimated values.

In the $25 \mathrm{~cm}$ ball mill grinding experiments reported in this paper, typically less than $1 \%$ material was lost in each experiment. Other researchers have reported experimental errors of magnitude of $3 \%$ in similar experiments. Hence, in the statistical analysis, an error of $5 \%$ in individual mass size fraction was assumed.

In the statistical analysis, which is fashioned after the usual Monte Carlo methods, the true value of mass fraction is colored with normally distributed error as follows:

$$
x=x *+\sigma N(0,1)
$$


where $x$ and $x *$ correspond to observed and true values of mass fractions, and $N(0,1)$ is the standard normal variate with zero mean and unit variance. Therefore, the observations are normally distributed with mean $x *$ and variance $\sigma^{2}$.

Numerous data sets were created by distributing normal random variate as in Eq. (10) over experimental observations of limestone dry grinding experiment with Feed A. Fig. 13 summarizes the statistical analysis of estimated breakage rates from these data sets. Breakage rates for three different size intervals are plotted with $a \pm 2$ standard deviation limit around them. For the sake of clarity in the figure, breakage rates for other size intervals are not shown. The vertical bars around the breakage rate for true data represent the range in which the values of estimated breakage rates will be with $95 \%$ confidence. Even though experimental errors cause estimation errors, all of the estimates fall around the true values in a random fashion. Furthermore, experimental errors do not cause unusual variations in the breakage rates with respect to time. In other words, the true trends are preserved by the G-H estimation scheme even in the presence of experimental errors.

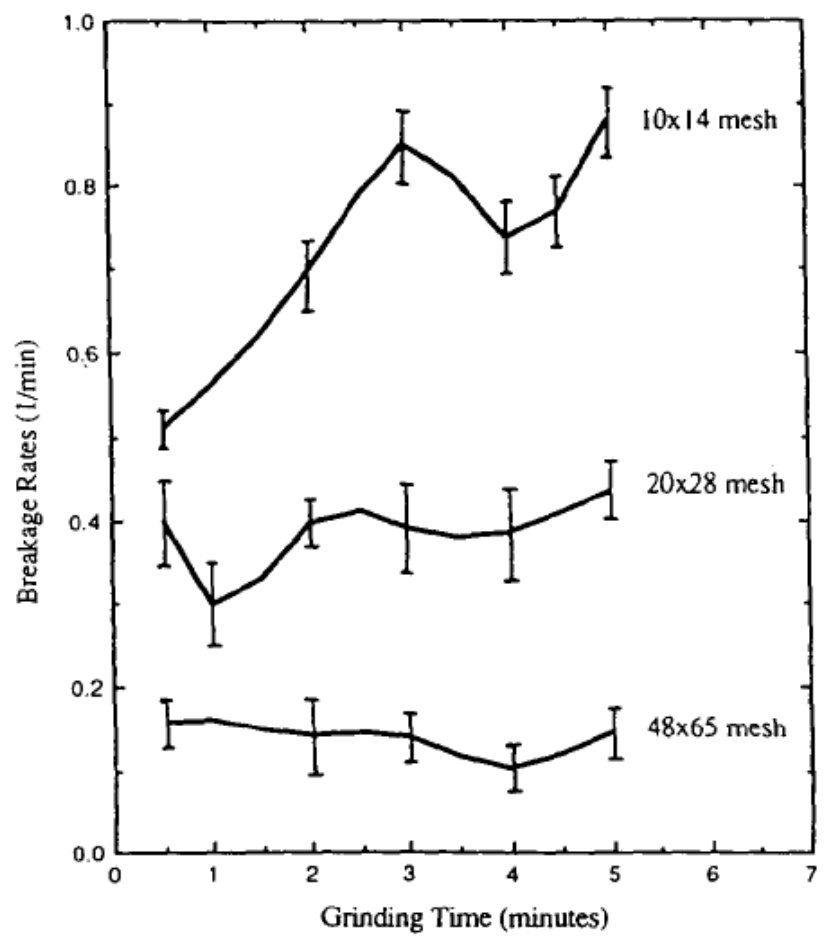

Fig. 13. Time dependence of breakage rates for a limestone dry grinding experiment. Vertical bars represent \pm 2 standard deviation range ( $95 \%$ confidence interval) around the estimated breakage rates in the presence of $5 \%$ error.

\section{Factors Affecting Breakage Rates in Batch Grinding}

Breakage of particles in ball mills is a complex process to analyze because both material properties and mill environment contribute to the evolution of size distribution. The energy consumed in the particle fracture which goes to the extension of cracks is primarily a function of material property. A detailed study of single particle and particle bed fracture under 
controlled loading is found in Schfnert [26]. However, in the ball mill, the multi-ball collisions produce a spectra of impact forces and therefore the fracture process cannot be analyzed from a fundamental point of view. Also, a number of environment factors contribute to the breakage. In instances where a single factor dominates the grinding environment, the consequence on particle size distribution is understood precisely. On the contrary, more than one factor is in effect inherent in wet grinding. A qualitative analysis of these factors is discussed in the following.

\section{Number of Contact Forces}

The number of contact forces influences the crack initiation, crack pattern, and the size, shape and number of fragments produced. The general tendencies [26] are the following: Breakage probability increases with the increasing number of contact forces. However, the probability of breakage decreases if more than about ten forces act at the same time from various directions. With more forces acting on a particle, more cracks run in the outer area of the particle, producing smaller fragments.

Since the number of contacts depends on the size distribution of particles present in the mill, the breakage rates change as grinding proceeds. In other words, continuous change in particle size distribution in the mill changes the number of contact forces around particles, which leads to acceleration and deceleration of breakage.

\section{Particle Size}

Particle strength and specific breakage energy vary among particles of the same size because of inherent variations in particulate properties [27]. When grinding begins, weak particles break first, leaving stronger particles behind which contribute to the acceleration of breakage in the beginning and deceleration of breakage as grinding time increases.

\section{Particle Size Distribution}

The size distribution of particles in the mill at any time affects the breakage rates of different sizes. Assume that the particles in the mill can be divided into two fractions: coarse and fine. The breakage rate of these particles will depend on the frequency of breakage events and on the energy consumed per unit mass. It has been experimentally verified by several researchers that, at any instant of time, the grinding zone is located at the toe of the ball charge in the tumbling mill. Coarse particles preferentially come to the toe position or to the grinding zone and hence are ground readily [28]. As the coarse fraction in the mill becomes very small, its grinding does not remain continuous in time, because the particles are very few in number and only some are caught in collisions with balls. Furthermore, when the coarse fraction in the mill becomes small, then each coarse particle will be surrounded by several layers of fines which can act as a cushion and absorb the impact force. The breakage rate of coarse particles decreases under these conditions. 
Viscosity

The viscosity of slurry in the mill increases due to the increased surface area of particles, which binds up water molecules and thus increases the effective solids concentration. Kawatra and Eisele [29] found that, at a constant solids content, a reduction in particle size produces an increase in slurry viscosity. Increase in viscosity can increase the probability of particle escape but reduce the energy and momentum of the grinding media. As two balls approach each other, the intervening slurry must be displaced. When viscosity is high, the particles in between the two approaching balls follow the streamlines of the escaping fluids and are not caught during the ball-ball impact. Therefore, it is extremely important to control the size distribution of the recycled material in closed-circuit grinding, because an excessive amount of fine material in recycling may increase slurry viscosity inside the mill beyond its critical value and thus reduce grinding efficiency.

\section{Settling of Particles}

During wet grinding, small particles tend to be suspended in the turbulent fluid medium, whereas larger particles settle to the bottom. This increases the probability of breakage of coarse particles and they are ground by the cascading ball motion [17].

\section{$\underline{\text { Grinding Additives }}$}

Chemical additives modify the flow of pulp in the grinding mill by changing the slurry viscosity $[13,30,31]$. This alters the mass-transfer process in the mill. In addition, a change in slurry viscosity may change the manner in which particles are distributed in the mill and get nipped by the moving balls. Grinding additives also influence the re-agglomeration of freshly produced fines by keeping the particles well dispersed and/or preventing fusion of particle surfaces. The strength of the material is affected by the chemical additive due to its effect on crack initiation and crack extension energy and on the retardation of rejoining or sealing of the freshly created cracks.

\section{Discussion and Conclusions}

Energy efficiency in the grinding process is of serious concern in the minerals industry; hence, a study of the rate of breakage of particles as a function of mill environment is useful. Analyzing how breakage rates change, if they do, can help improve grinding efficiency in several ways. It can help identify operating conditions that maximize either the mill throughput or the rate of size reduction within the mill. Analysis of mill environment-dependent breakage rates can help resolve the debate about lowering the hydrocyclone efficiency to improve the grinding-circuit performance [32]. In the event that breakage rate of coarse particles increases in the presence of fines, the hydrocyclone operation can be purposely set to recirculate some of the fines. Finally, incorporating mill environment-dependent breakage rates in mill scale-up and design will be very desirable. 
Until recently, it was believed that in dry grinding, breakage rates of particles in different size intervals did not change with grinding time. Analysis of dry grinding experiments performed with limestone in this study suggests that breakage rates are dependent on the instantaneous particle size distribution of powder in the mill. Particles in finer feed break at a higher rate than coarser feeds. Therefore, it is concluded that the presence of fines increases the rate of breakage of coarse particles.

In wet grinding, the viscosity of the slurry is changing continuously. At any instant in the ball mill, coarse particles are settling and the motion of the mill brings about remixing. Since a multiplicity of factors are in play at any given time it is impossible to delineate the factors causing acceleration or deceleration of particles.

Grinding aids primarily alter the viscosity of the slurry. At very high percent solids, say $80 \%$ or $85 \%$, the slurry tends to be incompletely wetted lumps and hence breakage is erratic. Upon adding the grinding additive XFS4272, the slurry regains its fluidity and the grinding rate becomes normal and orderly. However, there is no specific increase in grinding rates due to the grinding aid. Still, the minerals industry can benefit from the use of an additive because it improves the flow properties of charge inside the mill and enables grinding at higher slurry density.

\section{List of Symbols}

$\begin{array}{ll}b_{i j} & \begin{array}{l}\text { breakage function; fraction of particles broken out of size interval } j \text { reporting to } \\ \text { size interval } i\end{array} \\ B_{i, j} & \text { cumulative breakage distribution function } \\ G_{i} & \text { a parameter in an approximate solution of batch grinding equation } \\ H_{i} & \text { a parameter in an approximate solution of batch grinding equation } \\ K_{i} & \text { breakage rate of particles in size interval } i \\ m_{i}(t) & \text { mass of particles in size interval } i \text { at time } t \\ n & \text { total number of size intervals } \\ N(0,1) & \text { standard normal variate } \\ R_{i} & \text { cumulative mass fraction of particles retained over size ' } i \text { ' } \\ t & \text { time } \\ x, x * & \text { true and observed value of mass fraction used in Eq. (10) } \\ G r e k \text { letters } & \end{array}$

$\alpha_{1}, \alpha_{2}, \alpha_{3} \quad$ parameters in functional form for breakage distribution 
parameter in functional form for breakage rate

\section{Acknowledgement}

This work was supported by the Department of the Interior's Mineral Institute Program administered by the US Bureau of Mines through the Generic Mineral Technology Center for Comminution, under Grant No. GI105149.

\section{References}

[1] J.A. Herbst and D.W. Fuerstenau, Trans. SME, 241 (1968).

[2] J.A. Herbst and D.W. Fuerstenau, Trans. AIME, 254 (1973) 343.

[3] J.A. Herbst and D.W. Fuerstenau, Int. J. Miner. Process., 7 (1980) 1.

[4] V.K. Gupta and P.C. Kapur, Powder Technol., 10 (1974) 217.

[5] V.K. Gupta, 1st World Particle Technology Conf., Part H-Comminution, 6th Eur. Syrup., Nuremberg Germany, 1986.

[6] V.K. Gupta, SME-AIME Ann. Meet., Denver, CO, USA, 1987, Prepr. No. 87-152.

[7] D.W. Fuerstenau and A.M. Abouzeid, Int. Z Miner. Process., 21 (1991) 151.

[8] C. Tangsathitkulchai and LG. Austin, Powder Technol., 56 (1988) 293.

[9] C. Tangsathitkulchai and L.G. Austin, Powder Technol., 59 (1989) 285.

[10] L.G. Austin, R.R. Klimpel and P.T. Luckie, Process Engineering in Size Reduction: Ball Milling, SME Publications, Littleton, CO, 1984.

[11] R.R. Klimpel, SME Ann. Meet., Salt Lake City, UT, USA, 1990.

[12] R.R. Klimpel, Miner. Met. Process., 8 (1991) 73.

[13] R.R. Klimpel and L.G. Austin, Powder Technol., 31 (1982) 239.

[14] R.R. Kiimpel and W. Manfroy, Ind. Eng. Chem. Process Des. Dev., 17 (1978).

[15] P.C. Kapur, Miner. Met. Process., 5 (1988) 133.

[16] K.J. Reid, Chem. Eng. Sci., 20 (1965) 53.

[17] J.A. Herbst, D.Eng. Dissertation, University of California at Berkeley, 1971.

[18] K. Rajamani and J.A. Herbst, Trans Inst. Min. Metall. (Sect C: Miner. Process. Extr. Metall.), 93 (1984).

[19] P.C. Kapur, Powder TechnoL, 33 (1982) 269.

[20] P.C. Kapur, Trans. Soc. Min. Eng. AIME, 247 (1970) 299. 
[21] P. Purker, R. Agrawal and P.C. Kapur, Powder Technol., 45 (1986) 281.

[22] R.K. Ralamani and D. Guo, Int. Z Miner. Process., 34 (1992) 103.

[23] R. Verma, M.S. Thesis, University of Utah, 1993.

[24] W.H. Press, B.P. Flannery, S.A. Teukalsky and W.T. Vetterling, Numerical Recipes, Cambridge University Press, Cambridge, 1988.

[25] M. Siddique, M.S. Thesis, University of Utah, 1977.

[26] K. Schfnert, 4th Tewksburg Symp., Melbourne, Australia, 1979.

[27] F. Bourgeois, R.P. King and J.A. Herbst, Comminution: Theory and Practice, SME Publications, Littleton, CO, 1992, p. 99.

[28] P.B. Phatak, M.S. Thesis, University of California at Berkeley, 1990.

[29] S.IC Kawatra and T.C. Eisele, Int. J. Miner. Process., 22 (1988) 251.

[30] H. El-Shell and P. Somasundaran, Powder Technol., 38 (1984) 275.

[31] H. El-Shell and P. Somasundaran, Powder Technol., 38 (1984) 267.

[32] D.D. Schlepp and P.A. Turner, SME Ann. Meet., Salt Lake City, UT, USA, 1990. 